\title{
Research on the Classification of High Resolution Image Based on Object-Oriented and Class Rule
}

\author{
Li Chaokui ${ }^{1,2}$, Fang Jun ${ }^{1,2}$, Wu Baiyan ${ }^{1,2}$ and Chen Jianhui ${ }^{1,2}$ \\ 1. National-Local Joint Engineering Laboratory of Geo-Spatial Information Technology, Hunan University of Science and \\ Technology, Xiangtan 411201, Hunan, China
}

2. Hunan province Engineering Laboratory of Geo-Spatial Information Technology, Hunan University of Science and Technology, Xiangtan 411201, Hunan, China

\begin{abstract}
With the development of remote sensing technology, the spatial resolution, spectral resolution and time resolution of remote sensing data are greatly improved. How to efficiently process and interpret the massive high resolution remote sensing image data for ground objects, which are of spatial geometry and texture information, has become the focus and key issue in the field of remote sensing research. A new method of the classification (OCRC (object-oriented and class rule classification)) of remote sensing, which is of object-oriented and rule, has been presented in this paper, that is, through the discovery and mining the knowledge of spectrum and spatial characteristics of high-resolution remote sensing image, establish a multi-level network image object segmentation and classification structure of remote sensing image to achieve classification and accuracy assessment accurately and quickly for ground targets. Selected the worldview-2 image data in the Zangnan area as a study object, using the OCRC to verify the experiment which was a combination of the mean variance method, the maximum area method and the accuracy comparison to analysis selected three kinds of optimal segmentation scale and established a multi-level image object network hierarchy for image classification experiments. The results show that the OCRC can enable the high resolution image classification results similar to the visual interpretation of the results, and has higher classification accuracy. The overall accuracy and Kappa coefficient of the object-oriented rule classification method are $97.38 \%, 0.9673$; compared with object-oriented SVM method, respectively higher than $6.23 \%, 0.078$; compared with object-oriented KNN method, respectively more than $7.96 \%, 0.0996$. The extraction precision and user accuracy of the buildings compared with object-oriented SVM method, respectively higher than $18.39 \%, 3.98 \%$, respectively better than the object-oriented KNN (K-Nearest Neighbor) method 21.27\%, 14.97\%.
\end{abstract}

Key words: Object-oriented, rules, high resolution, multi-scale segmentation.

\section{Introduction}

With the spatial resolution, spectral resolution and time resolution of remote sensing data improved significantly, deeply mining and using all kinds of semantic association of remote sensing data, removing the interference factors effectively and improving the automation and accuracy of interpretation has become the main challenge of the development and application of high resolution remote sensing [1]. The method of object-oriented classification [2-4] overcomes the defects of traditional high-resolution remote sensing

Corresponding author: $\mathrm{Li}$ Chaokui, Ph.D., professor, research fields: spatial information modeling and its application. image classification. Not only make full use of a variety of knowledge extraction rules as the basis of the high complexity and difference of the high-resolution image classification, and integrate the segmentation and classification to some extent, so as to realize the rapid and accurate classification of remote sensing images, improve the precision and speed of image classification. The research based on the remote sensing data of Worldview-2 Cuona County Zangnan area, uses the OCRC method to classify and extract the information of features effectively and accurately [5-7]. On this basis, a new method of OCRC (object-oriented and class rule classification) of high resolution image classification 
has proposed in this paper. The OCRC by the discovery and excavating the rich knowledge of spectral and spatial characteristics of high-resolution spectral image, establishing image object segmentation and classification structure at multiple levels of network, the classification results and object-oriented nearest neighbour classification method classification results were compared and analyzed. The results show that the OCRC proposed in this paper has obvious advantages in the image building extraction accuracy and user accuracy.

\section{Information Extraction Methods}

\subsection{Study Area and Data Sources}

The study area is located in the Cuona county of southern Tibet. Southern Tibet is the northern tropical area in the world, with warm and humid climate, plentiful hydrothermal conditions, it is said that study area is the "south of Yangtze River" of Tibet. It is the most complete altitudinal belts in parts of the world, one of the most abundant water resources in the region of China. The main rivers Brahmaputra whole basin water reserves after the Yangtze River and the whole basin water reserves of unit area and Long River is the river in China for the first time. Using the November 28, 2012 Worldview-2 remote sensing image as data source. Worldview-2 image includes $0.5 \mathrm{~m}$ panchromatic and 1.8 resolution multi spectral bands, with four standard spectrum (red, green, blue and near infrared). The pre-treatment of remote sensing images includes geometric correction, image fusion and image cropping.

\subsection{The Best Segmental Scale}

The phenomenon and process of surface entities are objectively true, and the phenomenon and process of research objects with different scales can show the complexity of surface entity [8]. On an optimal scale, clearly study and observing the research object geographical phenomenon, processes and the rules of geographical features, maintain the spatial structure characteristic consistent to the size of entity objects that is the best segmental scale of features, which affect the classification results and accuracy directly. Generally, the method of select best segmentation scale is relatively single, and not comprehensive analysis of the image characteristics, which may result in the selection of segmentation scale is not accurate, affecting the classification accuracy. Therefore, to improve the single segmentation method, the mean variance method, the maximum area method [9], the precision comparison method were integrated, used these three methods to analysis best segmentation scale respectively and compared the various optimal scales, to overcome the one-sided nature of a single method. The best segmentation scales for various features of three kinds of methods were selected, and established a multi-level image object network hierarchy for image classification to achieve the optimal segmentation scale. This experiment selects the scales of $10,20,30,40,50,60,70,80,90,100$, 110 to partition experimental area. The results of the experiments presented, the optimal segmental scale of mean-variance method is $30,60,80$, the optimal segmental scale interval of maximum area method is $(40,50),(60,80),(90,100)$. And the segmental scale of precision comparison method in buildings, water, vegetation, bare land, road, at respectively 30, 80, 60, 20,30, the classification accuracy reached maximum value (as shown in Figs. 1-3). In conclusion, choose the segmentation scale of 80 to distinguish water and non-water, the segmentation scale of 60 to distinguish vegetation and non-vegetation in non-water, the segmentation scales of 30 to distinguish buildings, roads and bare land in non-vegetation.

\subsection{Analysis of Spectral and Spatial Features of Ground Objects}

\subsubsection{Spectral Imprints}

Through the overall observation and analysis of the images, in this paper, the study area is divided into five features, that is vegetation, water, roads, bare land and building, select and observe how changes in the 


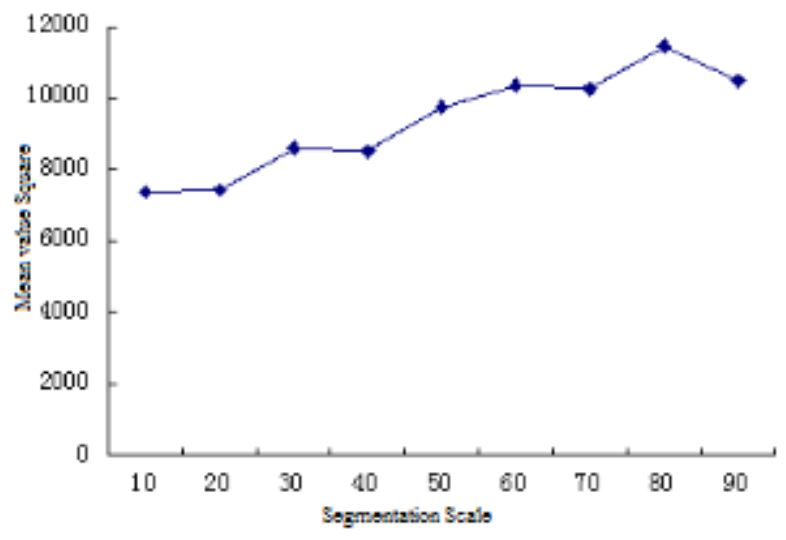

Fig. 1 Relationship between segmentation scales and mean-variance.

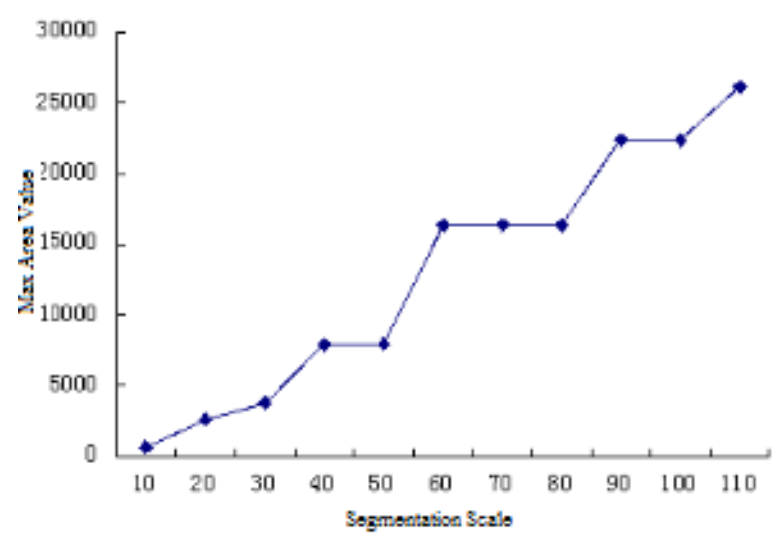

Fig. 2 Relationship between segmentation scales and maximum area values.

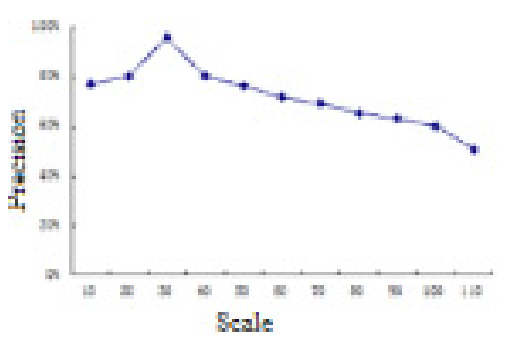

(a) Building

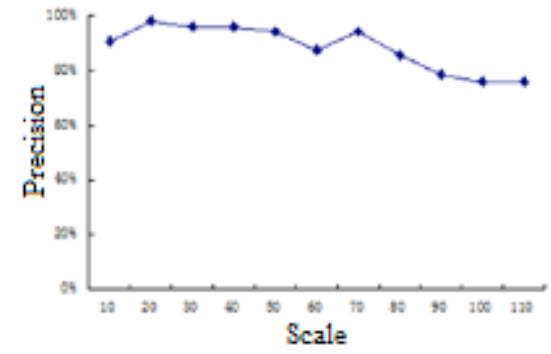

(d) Bare land

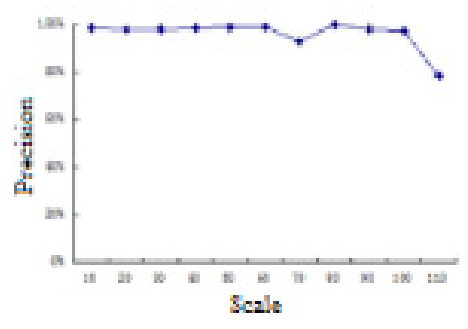

(b) Water

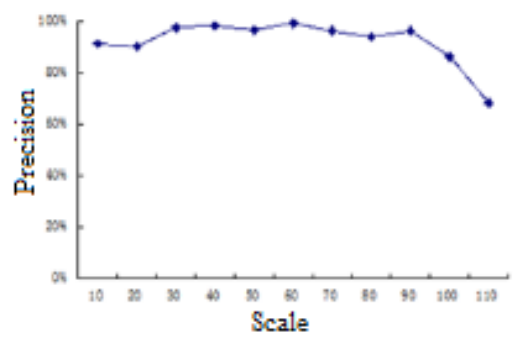

(c) Vegetation

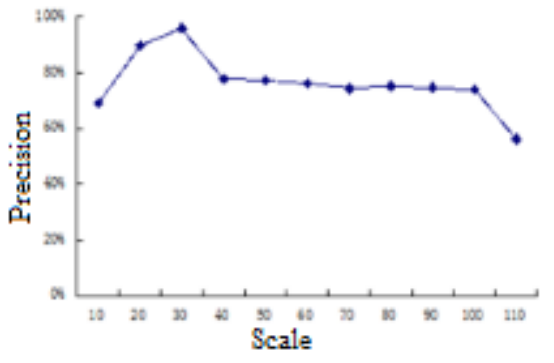

(e) Roads

Fig. 3 Relationship between scale and precision.

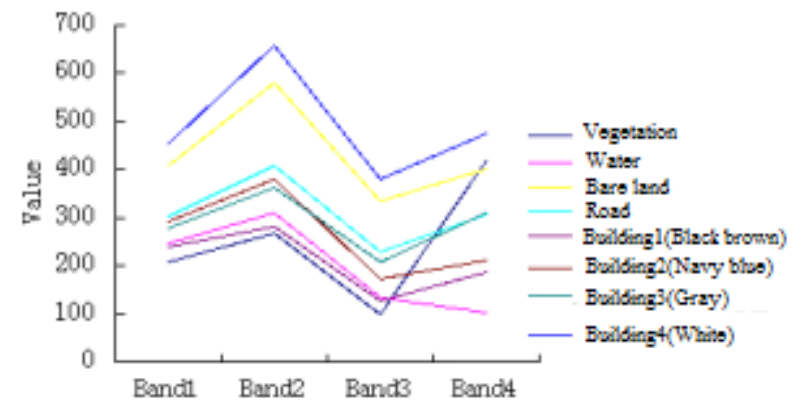

Fig. 4 Terrain characteristic spectrum curve.

image spectral characteristics of these five samples (shown in Fig. 4), and summarize their characteristics. Finally, according to vegetation, water, roads, bare land and building, 30 sizes for 10-15 typical regional pixel samples were selected respectively on the image. Experiment results show that the separation between each object class of training samples was greater than 0.8 , separation is better and more suitable for the spectral analysis as the training sample.

By collecting and analyzing a large number of characteristics and changes of the typical values of ground object features, using the spectral correlation relationship, summarize some outburst feature and distribution law, so that it can fully reflect the attributes and spatial distribution of the surface of objects. Give help for image classification and improve classification accuracy. 
Table 1 Object classification rules of research area.

\begin{tabular}{|c|c|c|}
\hline Scale layer & Surface features & Rules \\
\hline First layer & Water bodies & $\begin{array}{l}230 \leq \text { Mean }(\text { Blue }) \leq 320 ; \\
0.2 \leq \text { Mean }(\text { NDWI }) \leq 0.6 ; \text { area } \geq 30,000\end{array}$ \\
\hline \multirow[t]{3}{*}{ Second layer } & Vegetation & $\begin{array}{l}\text { Mean(NDVI) } \geq 0.36 \\
200 \leq \text { Mean }(\text { Green }) \leq 500 ; 0.6 \leq \max . \operatorname{diff} \leq 1.8\end{array}$ \\
\hline & Bare land & $\begin{array}{l}305 \leq \text { Brightness } \leq 500 \\
0.03 \leq \text { Mean }(\text { NDVI }) \leq 0.19 ; 450 \leq \text { Mean }(\text { Green }) \leq 640\end{array}$ \\
\hline & Roads & $\begin{array}{l}300 \leq \text { brightness } \leq 600 ; \mathrm{F}=\mathrm{B}-\mathrm{NIR}<0 ; \\
\text { length/width } \geq 4.5 ; 8 \leq \text { width } \leq 25 ; \text { Mean }(\text { Green })<450 ; \\
0.3 \leq \text { density } \leq 1.5 ; 2 \leq \text { Curvature/length } \leq 6 ;\end{array}$ \\
\hline Third layer & Buildings & $\begin{array}{l}F=B-N I R \geq 0 \\
\text { Rectangular fit } \geq 0.78 \\
200 \leq \text { Mean }(\text { Blue }) \leq 278 \\
\text { Mean }(\text { Green })>640\end{array}$ \\
\hline
\end{tabular}

The NDVI values of the vegetation are very different with other ground feature categories. The NDVI values of vegetation are the highest in all types, the average value is greater than 0.6 , while the average NDVI value of the other land is less than 0.2 in Fig. 5a. The NDVI values of water bodies and other surface features are significantly different, which are shown as white in the result picture, and the range of the water body index is usually between $[0.29,0.6]$ (Fig. 5b). Therefore, NDWI and NDVI can be used to distinguish vegetation and water. The typical spectral characteristics of surface features showed in Fig. 4, the brightness value of roads and building is both: Band $2>$ Band $1>$ Band $4>$ Band 3. In order to separate the roads and the building, the $\mathrm{F}=$ (B1-B4)/B1 is greater than the threshold that value 0 is for the building while the threshold value less than or equal to 0 is for the roads, which were obtained by the spectral characteristics analysis and repeated tests. In the Worldview-2 images, while the characteristics of bare land, roads and buildings are quite obvious which can be distinguished by visual, however, because the order of magnitude to the brightness mean value changes of the bare land, the part of roads and the buildings (white) is similarity, and those were showed white in the image spectra. Therefore, in order to distinguish the bare land, the part of roads and white building, that the threshold value of $B 2$, when greater than 640 is for the buildings, less than or equal to 640 and greater than or equal to 450 is for the bare land, and less than the 450 is for the road.

\subsubsection{Spatial Features}

Shape feature is a very important feature of target recognition and classification in high resolution image. Object-oriented classification has the object as the basic classification unit, the shape features of objects can be scientifically calculated with mathematical tools, artificial intelligence and nonlinear systems, thus it can accurately express and describe the shape of the real surface features. The study will choose length width ratio feature, width feature, rectangle suitability, curvature, standard deviation of curvature and density characteristics as the classification rules. The scale of 30 segmentation results as shown in Fig. 6.

\subsection{Classification Rules and Hierarchy}

After image segmentation, high-resolution image features with high complexity and difference are extracted by way of different knowledge of characteristics. Through the transfer between different levels to make the hierarchy have the inheritance relationship, constitute complex intellectual structure network of rules, to achieve the hierarchical classification of various features in the image highly efficient and accurate. This paper takes eCognition 8.7 


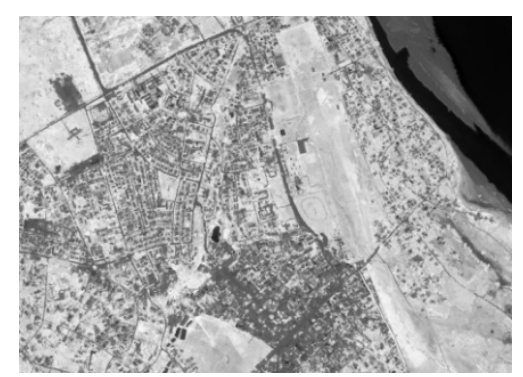

(a) Image of NDVI characteristic

Fig. 5 NDVI, NDWI image feature.

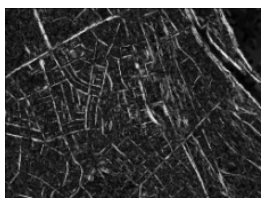

(a) Image of aspect ratio feature

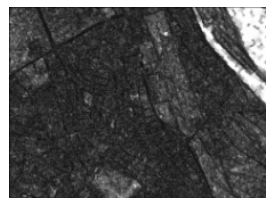

(b) Image of width feature

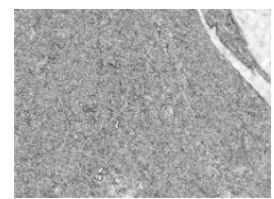

(c) Image of suitability for rectangle
Fig. 6 Results of all kinds of shape features.

as the platform, comprehensive utilization of spectral, shape, texture and context information of the each level object category in the image, select the appropriate classification feature or combination, set up classification rule set of all kinds of surface features. Based on the knowledge analysis and feature mining in the study area, we establish the hierarchical system and classification rules of water, vegetation, buildings, roads and bare lands, and classify Worldview-2 images step by step (Table 1).

\section{Analysis of Experimental Results}

The comparison of the reference for object inspection sample of known classification and the specific objects in classification images is carried out by experiments. Using the confusion matrix for accuracy evaluation method to calculate the user accuracy, producer accuracy, total precision and Kappa coefficient of the classification results, and the results of image information extraction are evaluated. Fig. $7 \mathrm{~b}$ and Table 3 are the object-oriented and class rules, classification results and classification accuracy evaluation form. The OCRC overall classification accuracy is $97.38 \%$ and kappa coefficient is 0.9673 , the producer and user accuracy of building are as high

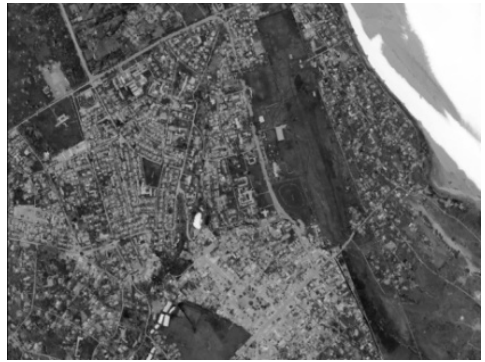

(b) Image of NDWI characteristic

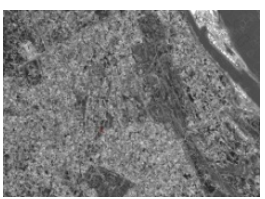

(d) Image of curvature

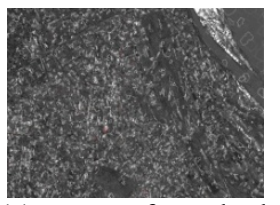

(e) Image of standard deviation of curvature

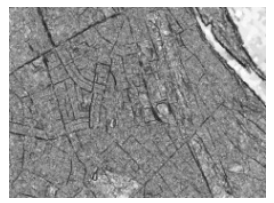

(f) Image of density characteristic as $91.67 \%$ and $96.37 \%$ respectively. The result of experiment shows that the OCRC is better. It can effectively distinguish the roads, bare land, buildings, which have similar spectral information, integrally extract all types of surface features information. By finding and mining characteristic knowledge of five kinds of surface features, select object features that are the same with the object-oriented and class rules for SVM and KNN, and a total of 20 feature indices are selected: spectra, shape, vegetation index, water index, etc. (Table 2). Figs. $7 \mathrm{c}$ and $7 \mathrm{~d}$, Tables 4 and 5 are

Table 2 Total of image objects features.

\begin{tabular}{lll}
\hline Category & Image object features & Quantity \\
\hline Spectrum & Mean & 4 \\
& Standard deviation & 4 \\
& Brightness & 1 \\
Shape & Max-diff & 1 \\
& Area & 1 \\
& Length & 1 \\
& Width & 1 \\
& Length/width & 1 \\
& Asymmetry & 1 \\
& Density & 1 \\
& Rectangular fit & 1 \\
Vegetation index & Shape index & 1 \\
Water index & NDVI & 1 \\
Total & NDWI & 1 \\
\hline
\end{tabular}


Table 3 Accuracy assessment of the OCRC results.

\begin{tabular}{|c|c|c|c|c|c|c|}
\hline Class & Vegetation & Water & Bare land & Roads & Building & Total \\
\hline Vegetation & 360 & 0 & 0 & 0 & 0 & 360 \\
\hline Water & 0 & 365 & 0 & 0 & 1 & 366 \\
\hline Bare land & 0 & 0 & 353 & 0 & 19 & 372 \\
\hline Roads & 0 & 0 & 6 & 352 & 9 & 367 \\
\hline Building & 0 & 3 & 1 & 8 & 319 & 331 \\
\hline Total & 360 & 368 & 360 & 360 & 348 & 1,796 \\
\hline Producer accuracy & 100.00 & 99.18 & 98.06 & 97.78 & 91.67 & \\
\hline User accuracy & 100.00 & 99.73 & 94.89 & 95.91 & 96.37 & \\
\hline
\end{tabular}

Overall accuracy $=97.38 \%$, Kappa $=0.9673$.

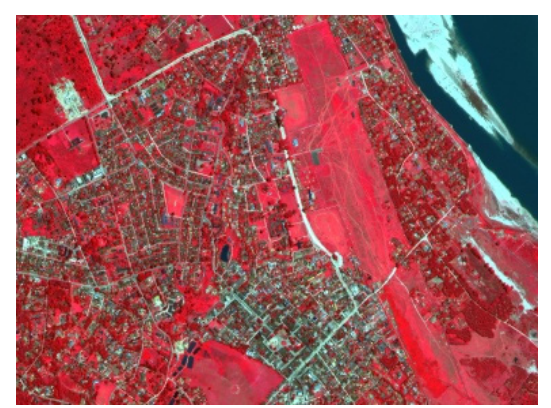

(a) Images in the study area

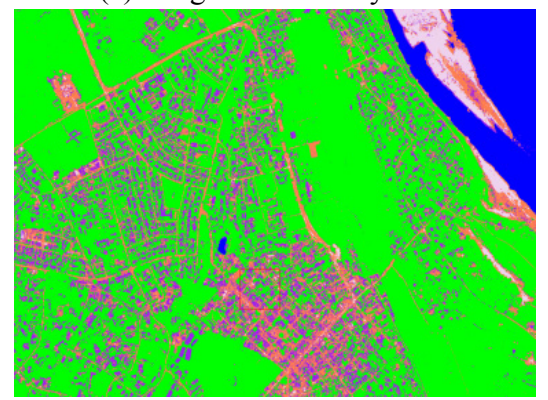

(c) Result of the object-oriented SVM classification

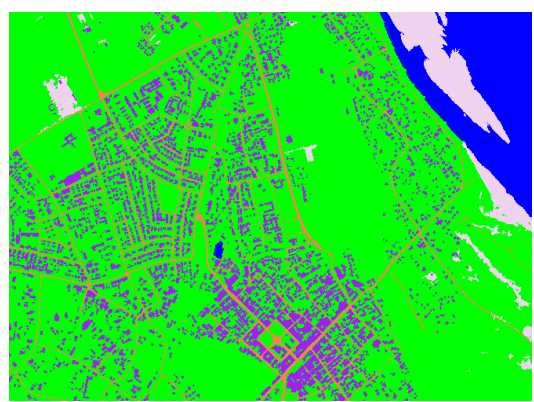

(b) Result of the OCRC

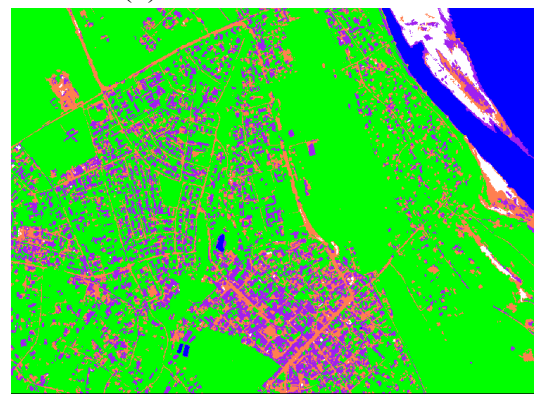

(d) Result of the object-oriented KNN classification

Fig. 7 Classification results of three methods.

Table 4 Accuracy assessment of the object-oriented SVM results.

\begin{tabular}{ccccccc}
\hline Class & Vegetation & Water & Bare Land & Roads & Building & Total \\
\hline Vegetation & 360 & 0 & 0 & 0 & 0 & 360 \\
Water & 0 & 343 & 0 & 0 & 0 & 343 \\
Bare Land & 0 & 0 & 343 & 16 & 60 & 419 \\
Roads & 0 & 19 & 10 & 336 & 33 & 398 \\
Building & 0 & 6 & 7 & 8 & 255 & 276 \\
Total & 360 & 368 & 360 & 360 & 348 & 1796 \\
Producer Accuracy & 100.00 & 93.21 & 95.28 & 93.33 & 73.28 & \\
User Accuracy & 100.00 & 100.00 & 81.86 & 84.42 & 92.39 & \\
\hline
\end{tabular}

Overall Accuracy $=91.15 \%$, Kappa $=0.8893$ 
Table 5 Accuracy assessment of the object-oriented KNN results.

\begin{tabular}{ccccccc}
\hline Class & Vegetation & Water & Bare Land & Roads & Building & Total \\
\hline Vegetation & 360 & 0 & 0 & 0 & 0 & 360 \\
Water & 0 & 368 & 0 & 0 & 12 & 380 \\
Bare Land & 0 & 0 & 320 & 24 & 44 & 388 \\
Roads & 0 & 0 & 7 & 313 & 47 & 367 \\
Building & 0 & 0 & 33 & 23 & 245 & 301 \\
Total & 360 & 368 & 360 & 360 & 348 & 1796 \\
Producer Accuracy & 100.00 & 100.00 & 88.89 & 86.94 & 70.40 & \\
User Accuracy & 100.00 & 96.84 & 82.47 & 85.29 & 81.40 & \\
\hline
\end{tabular}

Overall Accuracy $=89.42 \%$, Kappa $=0.8677$

classification results and accuracy evaluation respectively of the object-oriented SVM classification [9-11] and the object-oriented KNN classification.

\section{Conclusion}

A new remote sensing image classification method based on object-oriented and rule is proposed. The OCRC, object-oriented SVM classification method and object-oriented KNN classification method have been compared with and the results have been analysed. The research and experiment results show that:

(1) Object oriented classification method can very effectively use the spatial information characteristics of the true surface features, such as area, shape, length, aspect ratio, suitability for rectangle, and to take part in the images classification process, especially for the object types that are metameric substance of same spectrum effect is obvious.

(2) Producer accuracy, user accuracy, overall accuracy, kappa coefficient of buildings, roads, bare land, water, vegetation that are concluded from the OCRC are higher than object-oriented SVM method and object-oriented KNN method. Among them: the total accuracy of OCRC was $97.38 \%$, respectively, higher than the object-oriented SVM classification method and object-oriented KNN classification method $6.23 \%, 7.96 \%$. The Kappa coefficient was
0.9673 , which were 0.078 and 0.0996 respectively higher than the object-oriented SVM classification method and object-oriented KNN classification method. The producer accuracy and user accuracy of buildings were $18.39 \%$ and $3.98 \%$ higher than that of the object-oriented SVM classification method, and $21.27 \%$ and $14.97 \%$ that is higher than the object-oriented KNN classification method.

(3) By using object-oriented multi-scale segmentation, the image objects that are similar with the physical size of the image features can be generated. The result and accuracy of this classification are more accurate than the conventional pixel-based classification method. Including: The OCRC through discovery and mining characteristic knowledge of true surface features on the image, establish the classification rules of features, and, the classification results arrived from OCRC are better than the object-oriented classification method of supervision, such as object-oriented SVM classification method and object-oriented $\mathrm{KNN}$ classification method. In addition, the OCRC has higher precision.

\section{Acknowledgements}

This paper is funded jointly by projects of the National Natural Science Foundation of China (41571374), the key research project of Hunan 
Education Ministry (No.16A070), Nature Science Joint Funding of Hunan province and Xiangtan Local (No.2017JJ4037).

\section{References}

[1] Chen, J. 2011. "Object-Oriented Classification of High Resolution Remote Sensing Image Based on Support Vector Machine and Granularity Computing." Science of Surveying and Mapping 2 (40): 128-34.

[2] Baatz, M., and Schape, A. 1999. "Object-Oriented and Multi-scale Image Analysis in Semantic Networks." In Proc. of the 2nd International Symposium on Operation Alization of Remote Sensing. August 16-20th. Enschede ITC.

[3] Baatz, M., and Schape, A. 2000. "Multiresolution Segmentation: An Optimization Approach for High Quality Multi-scale Image Segmentation.” Angewandte Geographische Information sverarbeitung XII: 12-23.

[4] Cao, Y. T. 2011. "Research on Land Use Classification Rule Set Based on QuickBird Satellite Data." Geography and Geographic Information Science 27 (6): 28-31.

[5] Ketting R. L. 1976. "Computer Classification of
Remotely Sensed Multispectral Image Data by Extraction and Classification of Homogeneous Object." IEEE Transactions on Geoscience Electronics 14 (1): 19-26.

[6] Yan, L. 2012. "The Extraction of High Resolution Remote Sensing Images Based on Rules.” Journal of Wuhan University (Information Science Edition) 37 (6): 636-9.

[7] Zhang, L. 2010. "Object-Oriented Subspace Analysis for Airborne Hyper-Spectral Remote Sensing Imagery." Neuro Computing 73 (4): 927-36.

[8] Yan, L. 2010. Remote Sensing Image Processing Experiment Tutorial. Wuhan: Wuhan University Press, 24-30.

[9] Huang, H. P. 2003. Study on the Scale Problem in Object Image Analysis. Beijing: Graduate University of Chinese Academy of Sciences, 74-82.

[10] Lv, Y. H. 2001. "Scale and Scale Conversion Method in Ecology." Acta Ecologica Sinica, 2096-105.

[11] Rego, L. F. G. 2007. "Automatic Land-Cover Classification Derived from High-Resolution IKONOS Satellite Image in the Urban Atlantic Forest in Rio de Janeiro (Brazil) by Means of an Object-Oriented Approach." Applied Remote Sensing for Urban Planning, Governance and Sustainability, 25-36. 